Supporting Information for:

\title{
A Facile and Practical Synthesis of Peracylated 4-Thio-D-ribofuranoses from D-Glucose
}

\author{
Zhi-Hua Sun and Bing Wang* \\ Department of Chemistry, Fudan University, 220 Handan Road, Shanghai 200433, China. \\ wangbing@fudan.edu.cn
}

Table of Contents

1. General Information. $\quad$.......................S2

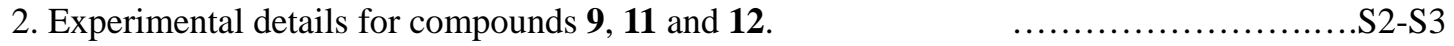

3. ${ }^{1} \mathrm{H}$ and ${ }^{13} \mathrm{C}$ NMR spectra for compounds $\mathbf{9}, \mathbf{1 1}, \mathbf{1 2}, \mathbf{1 3}, \mathbf{1 4}, \mathbf{1 5}, \mathbf{1 9}$.

4. ORTEP drawings for 9 and 14.

S18-S19 
General Information. All ${ }^{1} \mathrm{H}$ NMR and ${ }^{13} \mathrm{C}$ NMR spectra were recorded at ambient temperature in $\mathrm{CDCl}_{3}$ (93.94 kG, ${ }^{1} \mathrm{H} 400 \mathrm{MHz}$ ). Chemical shifts are reported in parts per million as follows: chemical shift, multiplicity $(\mathrm{s}=$ singlet, $\mathrm{d}=$ doublet, $\mathrm{t}=$ triplet, $\mathrm{q}=$ quartet, $\mathrm{m}=$ multiplet, $\mathrm{br}=$ broad), integration, and coupling constant. Optical rotations were recorded at $589 \mathrm{~nm}$, and were reported as $[\alpha]_{\mathrm{D}}$ (concentration in grams $/ 100 \mathrm{~mL}$ solvent). Melting points were uncorrected.

\section{5,6-Anhydro-3- $O$-benzoyl-1,2- $O$-isopropylidene- $\alpha$-L-allo-pentofuranose (9).}

To a stirred solution of mesylate $\mathbf{1 6}$ (4.95 g, $9.6 \mathrm{mmol}$ ) in $40 \mathrm{~mL}$ THF was added dropwise $1 \mathrm{M}$ TBAF in THF $(11.5 \mathrm{~mL})$ at rt. After $3 \mathrm{~h}$ the reaction was quenched by aq. $\mathrm{NH}_{4} \mathrm{Cl}$, THF was removed under reduced pressure and the residue extracted with EtOAc $(2 \times 60 \mathrm{~mL})$, the combined organic layer was washed with water and brine, dried $\left(\mathrm{Na}_{2} \mathrm{SO}_{4}\right)$, filtered and concentrated under reduced pressure to give 4.60 g yellow oil. To an ice-cold solution of the crude product in $20 \mathrm{~mL}$ DMF under Ar was added $\mathrm{NaH}$ (360 mg, 60\% in mineral oil, $9 \mathrm{mmol}$ ) in one portion under stirring. After 5 min the reaction was quenched by adding ice, the solution was diluted with EtOAc (100 $\mathrm{mL})$, washed with water and brine, dried $\left(\mathrm{Na}_{2} \mathrm{SO}_{4}\right)$, filtered and concentrated under reduced pressure. The residue was purified by silica gel column chromatography (EtOAc/hexane=1/6) to afford 9 (1.43 g, 51\%) as a white solid.

m.p. $123-124^{\circ} \mathrm{C} ;[\alpha]_{\mathrm{D}}{ }^{23}+120.3\left(c 0.74, \mathrm{CHCl}_{3}\right) ;{ }^{1} \mathrm{H}$ NMR $\left(\mathrm{CDCl}_{3}\right) \delta 8.06(\mathrm{~d}, 2 \mathrm{H}, J=7.0 \mathrm{~Hz})$, 7.60 (t, $1 \mathrm{H}, J=7.4 \mathrm{~Hz}$ ), 7.47 (t, 2H, $J=7.8 \mathrm{~Hz}$ ), 5.87 (d, $1 \mathrm{H}, J=3.5 \mathrm{~Hz}$ ), 5.01 (dd, $1 \mathrm{H}, J=8.6$, $4.7 \mathrm{~Hz}), 4.96(\mathrm{~m}, 1 \mathrm{H}), 4.20$ (dd, $1 \mathrm{H}, \mathrm{J}=8.6,4.3 \mathrm{~Hz}), 3.18(\mathrm{~m}, 1 \mathrm{H}), 2.88-2.80(\mathrm{~m}, 2 \mathrm{H}), 1.54$ (s, 3H), 1.33 (s, 3H); ${ }^{13} \mathrm{C} \mathrm{NMR}\left(\mathrm{CDCl}_{3}\right) \delta 165.6,133.5,129.8,129.1,128.5,113.4,104.5,77.5,77.4$, 73.8, 50.9, 43.8, 26.6. ESI-MS $m / z$ 307.1 $\left(\mathrm{M}+\mathrm{H}^{+}\right)$; HR-ESI-MS $m / z$ Calcd for $\mathrm{C}_{16} \mathrm{H}_{19} \mathrm{O}_{6}$ 307.1182, Found 307.1187.

\section{5,6-Anhydro-3-O-benzoyl-1,2- $O$-isopropylidene-5-thio- $\alpha$-D-gluco-pentofuranose (11).}

A solution of epoxide 9 (2.01 g, $6.57 \mathrm{mmol}$ ) and thiourea (524 mg, $6.9 \mathrm{mmol}$ ) in $\mathrm{MeOH}$ (25 $\mathrm{mL}$ ) under Ar was refluxed overnight. After cooling, the solvent was removed under reduced pressure and the residue partitioned between EtOAc and water. The organic layer is washed with water and brine, dried $\left(\mathrm{Na}_{2} \mathrm{SO}_{4}\right)$, filtered and concentrated under reduced pressure. The residue was purified by silica gel column chromatography (EtOAc/hexane=1/8 to 1/6) to afford 11 (1.473 g, 73\%) as an oil.

$[\alpha]_{\mathrm{D}}{ }^{23}+127.6\left(c\right.$ 1.19, $\left.\mathrm{CHCl}_{3}\right) ;{ }^{1} \mathrm{H}$ NMR $\left(\mathrm{CDCl}_{3}\right) \delta 8.09$ (d, $\left.2 \mathrm{H}, J=7.0 \mathrm{~Hz}\right), 7.60$ (t, $1 \mathrm{H}, J=$ $7.4 \mathrm{~Hz}$ ), 7.47 (t-like, 2H), 5.90 (d, 1H, $J=3.9 \mathrm{~Hz}$ ), 4.99 (t, $1 \mathrm{H}, J=3.7 \mathrm{~Hz}$ ), 4.94 (dd, $1 \mathrm{H}, J=8.6$, $5.1 \mathrm{~Hz}$ ), 3.96 (t-like, $1 \mathrm{H}$ ), 3.00 (m, 1H), 2.55 (dd, 1H, $J=6.3,1.2 \mathrm{~Hz}$ ), 2.32 (dd, 1H, $J=5.1,1.6$ $\mathrm{Hz}$ ), 1.52 (s, 3H), 1.32 (s, 3H); ${ }^{13} \mathrm{C}$ NMR $\left(\mathrm{CDCl}_{3}\right) \delta 165.8,133.4,130.0,129.3,128.5,113.2$, 104.1, 81.3, 77.6, 75.4, 32.6, 26.6, 22.0. ESI-MS $m / z$ 345.0 (M + Na $\left.{ }^{+}\right)$; HR-ESI-MS $m / z$ Calcd for $\mathrm{C}_{16} \mathrm{H}_{18} \mathrm{NaO}_{5} \mathrm{~S}$ 345.0773, Found 345.0770. 
3-O-Benzoyl-5,6-di-O,S-acetyl-1,2-O-isopropylidene-5-thio- $\alpha$-D-gluco-pentofuranose (12).

A mixture of 11 (1.33 g, $4.13 \mathrm{mmol})$, AcOK (1.08 g, $11.0 \mathrm{mmol})$ in $\mathrm{Ac}_{2} \mathrm{O}(10.8 \mathrm{~mL})$ and AcOH (2.3 mL) was refluxed for $36 \mathrm{~h}$. After cooling, the volatiles were removed under reduced pressure, the residue was taken up in EtOAc, washed with aq. $\mathrm{NaHCO}_{3}$ and brine, dried $\left(\mathrm{Na}_{2} \mathrm{SO}_{4}\right)$, filtered and concentrated under reduced pressure. The residue was purified by silica gel column chromatography (EtOAc/hexane=1/6) to afford $12(1.61 \mathrm{~g}, 92 \%)$ as an oil.

$[\alpha]_{\mathrm{D}}{ }^{23}+117.1\left(c\right.$ 1.48, $\left.\mathrm{CHCl}_{3}\right) ;{ }^{1} \mathrm{H}$ NMR $\left(\mathrm{CDCl}_{3}\right) \delta 8.08(\mathrm{~m}, 2 \mathrm{H}), 7.60$ (t-like, $\left.1 \mathrm{H}\right), 7.47$ (t-like, 2H), $5.86(\mathrm{~m}, 1 \mathrm{H}), 4.98-4.92(\mathrm{~m}, 2 \mathrm{H}), 4.48(\mathrm{~m}, 1 \mathrm{H}), 4.35(\mathrm{~m}, 2 \mathrm{H}), 4.07(\mathrm{~m}, 1 \mathrm{H}), 2.22(\mathrm{~s}$, 3H), 2.01 (s, 3H), 1.52 (s, 3H), 1.31 (s, 3H); ${ }^{13} \mathrm{C} \mathrm{NMR}\left(\mathrm{CDCl}_{3}\right) \delta 193.5,170.4,165.4,133.4$, 129.9, 129.2, 128.5, 113.2, 104.0, 77.7, 77.3, 74.8, 63.2, 43.6, 30.4, 26.7, 26.6, 20.6. ESI-MS $\mathrm{m} / \mathrm{z}$ 447.1 (M + Na ${ }^{+}$); HR-ESI-MS m/z Calcd for $\mathrm{C}_{20} \mathrm{H}_{24} \mathrm{NaO}_{8} \mathrm{~S}$ 447.1090, Found 447.1071. 

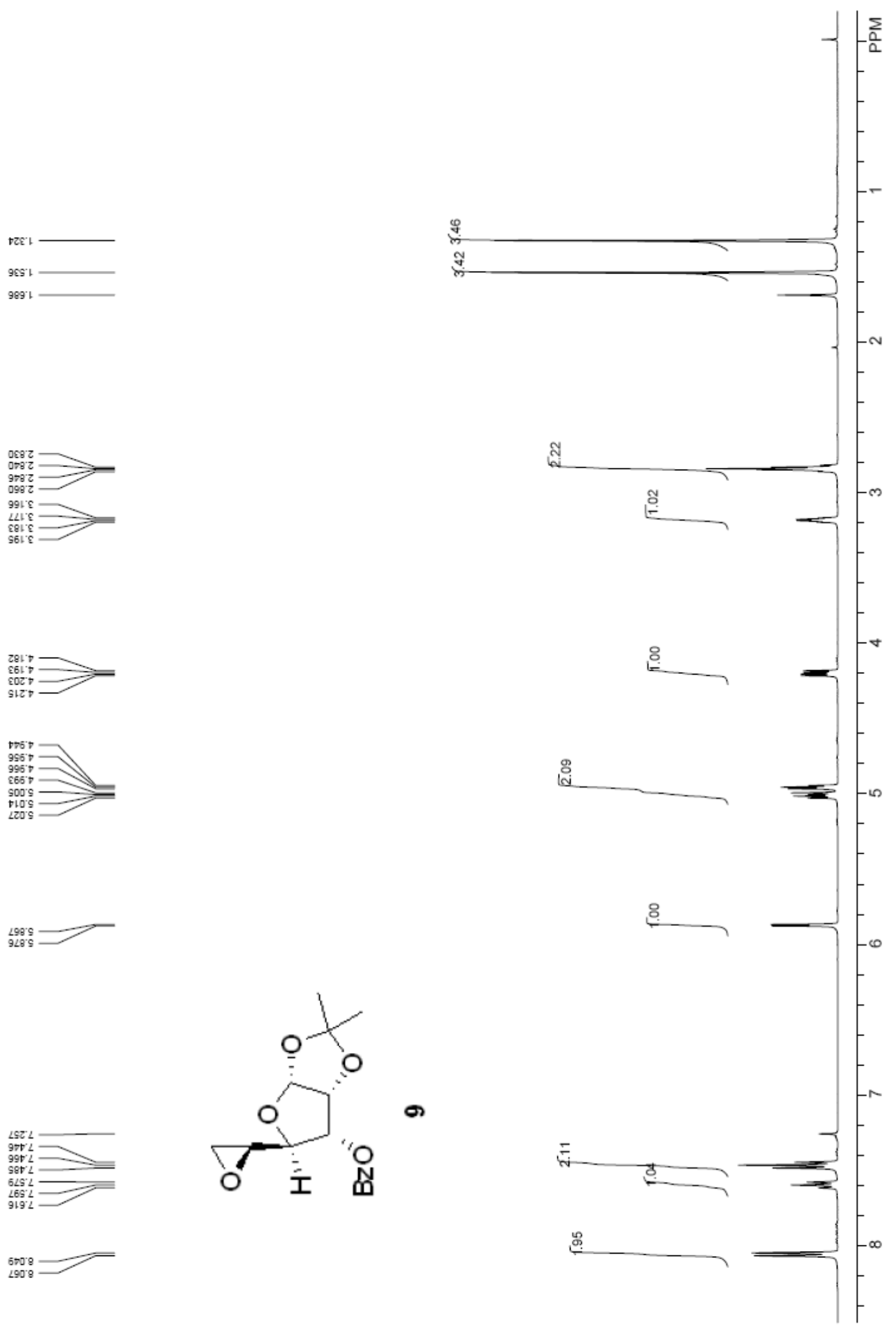
$289 \cdot 92-$

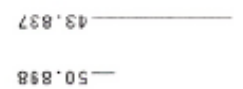

$800^{\circ} 82$
$089^{\circ} 92$

866.92

SIE. 2

I $90^{\circ} \angle L$

IZS'D0I

$29 \varepsilon^{\circ} 8 \mathrm{TI}$

$98 \mathrm{~V} \cdot 8 \mathrm{gt}$

$860^{\circ} 6 \mathrm{ZI}$

$96 L^{\circ} 6 \mathrm{ZI}-$
S8t

८ะ9.ร9I

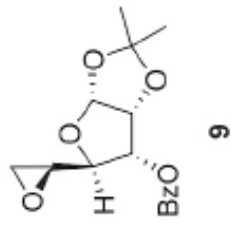

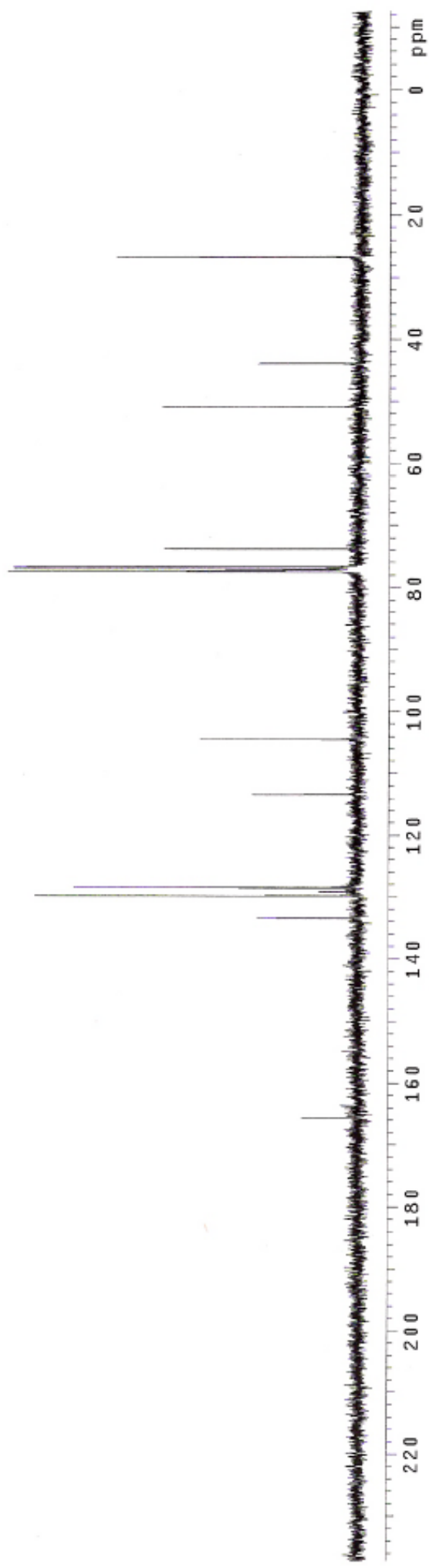




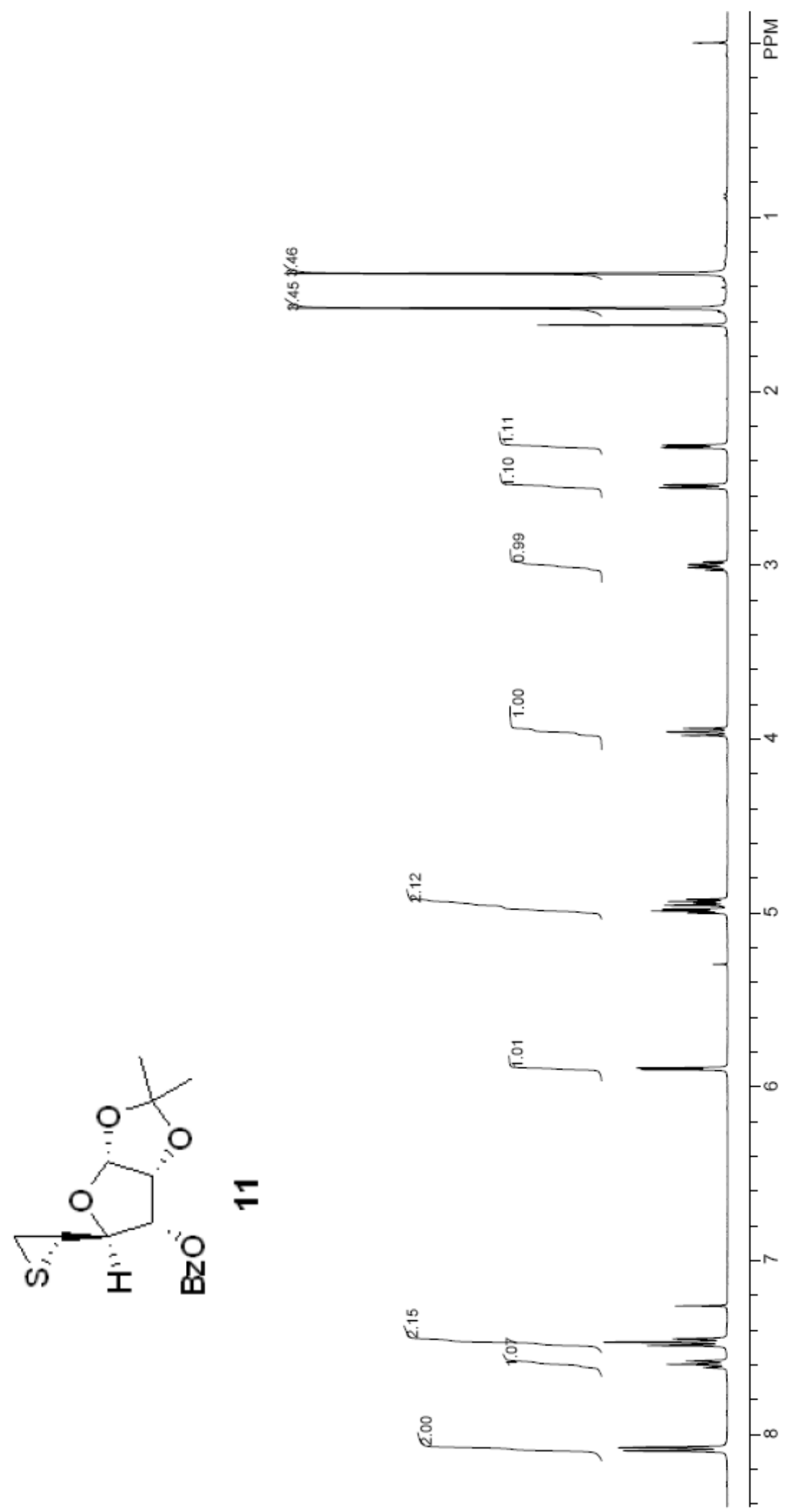

$686 \varepsilon$
$\angle 96 \varepsilon=$
$646 \varepsilon$

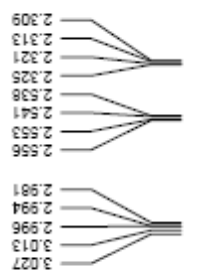

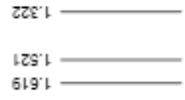

$126^{\circ} t$

${ }_{\varepsilon 76}^{+867}=$

$826^{\circ}$,

$886^{\prime} '-$
$000^{\prime} \mathrm{S}$

7689

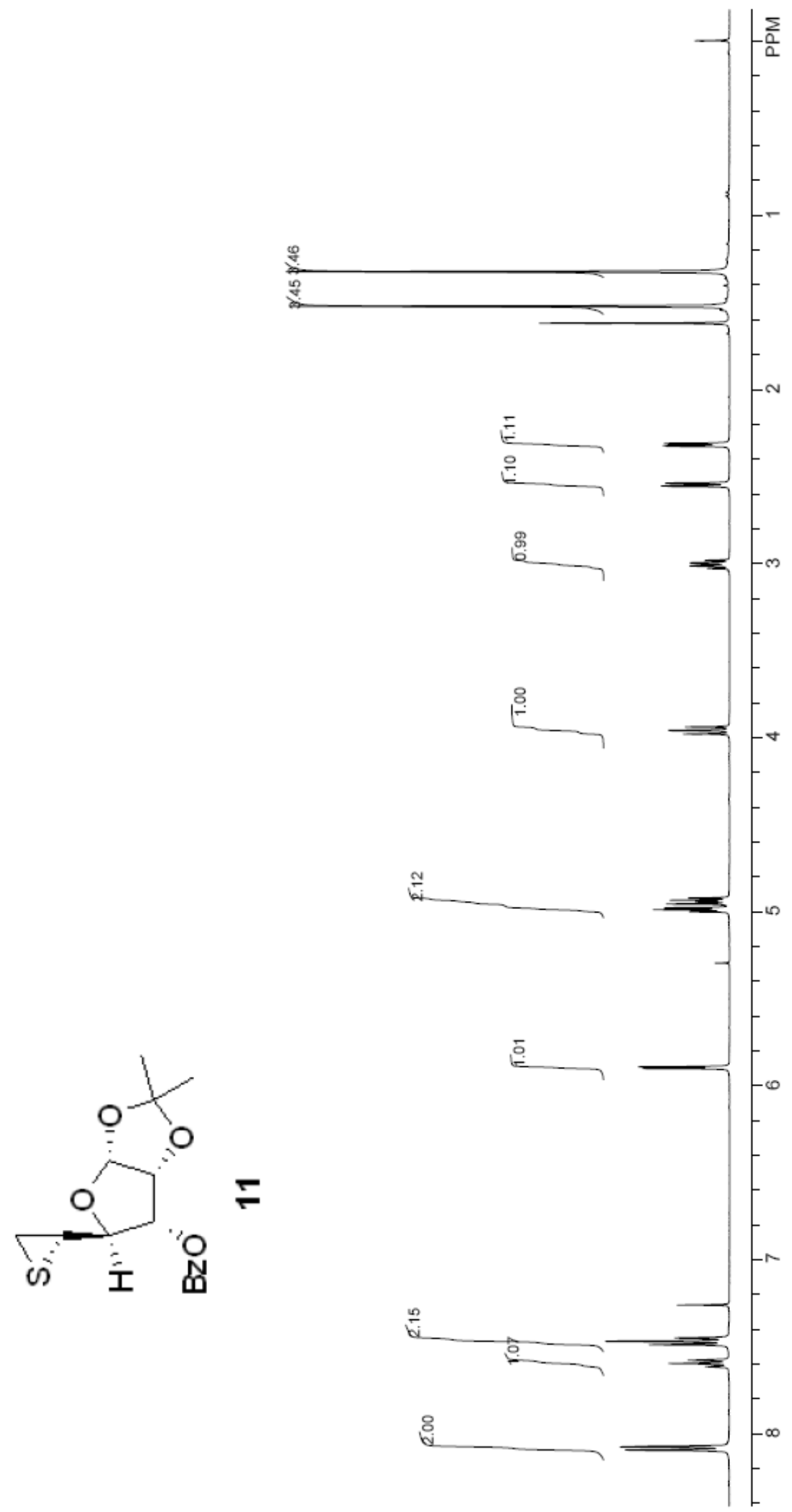

${ }_{6062}^{002} 2$

${ }_{800}^{200} 2$

${ }_{9652} \angle S L=$

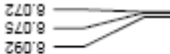



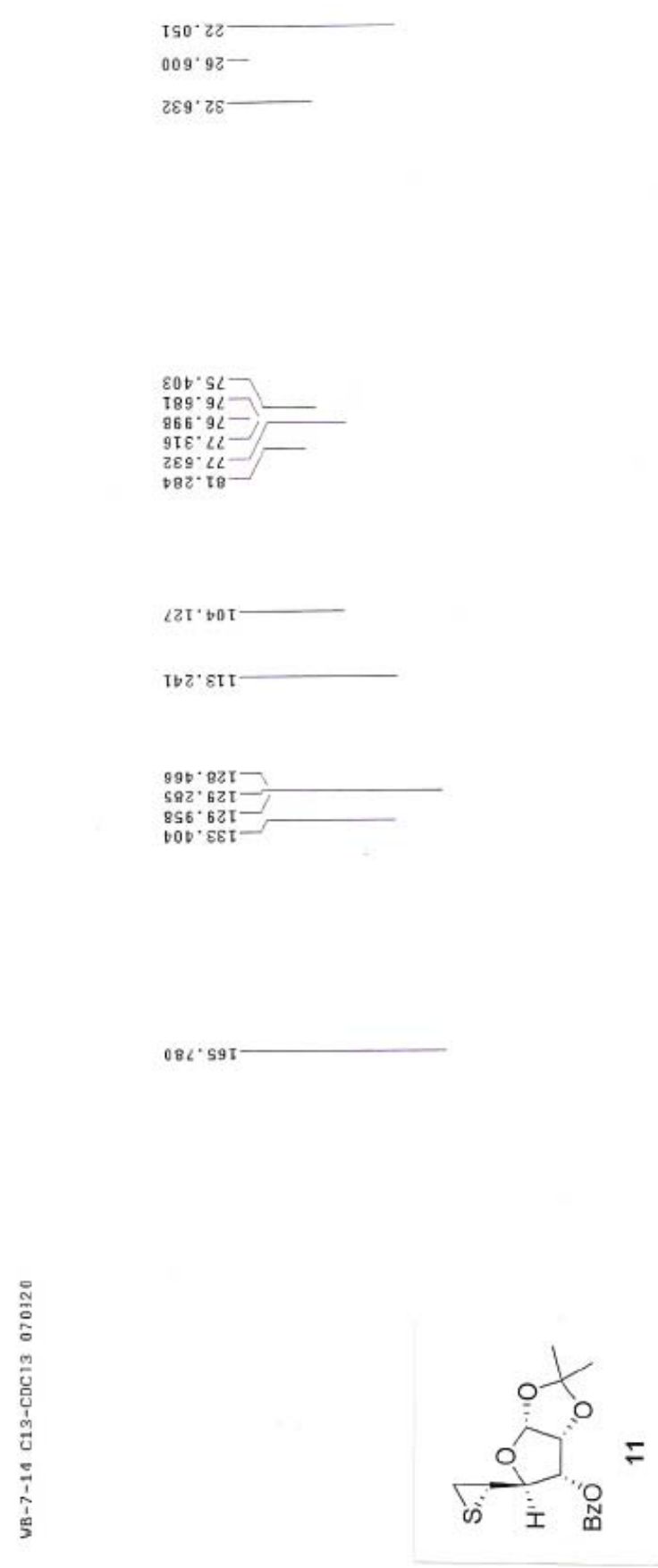

S7

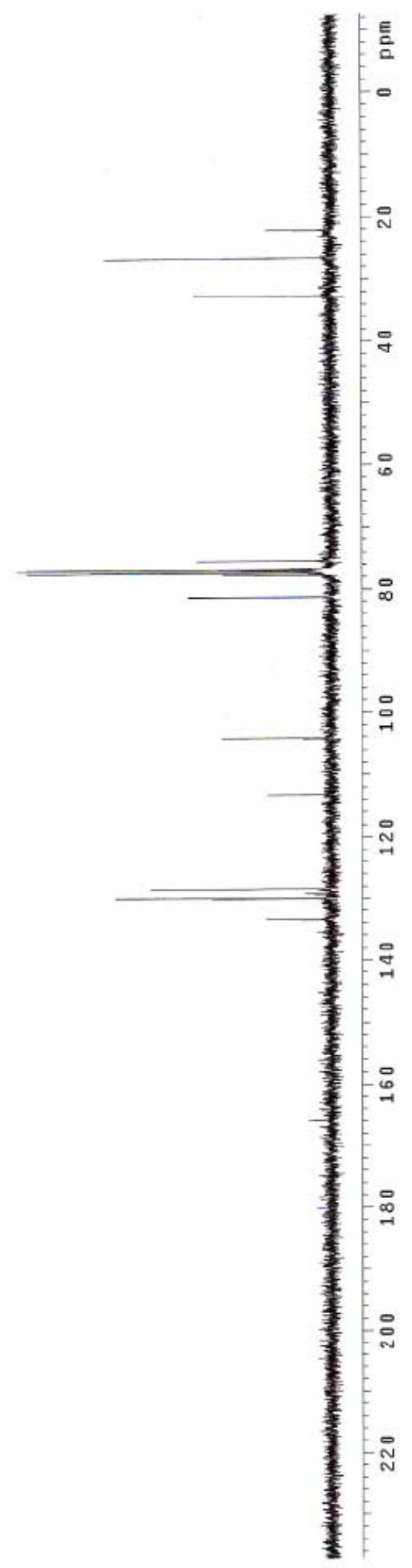




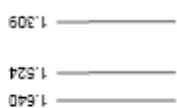

$600 \mathrm{Z}$

${ }_{9120}^{6002}$

$\varepsilon, 0,0$
$\angle S O D$

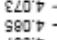

z\&: $D$

=

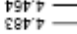

$\left.186^{\circ}\right\rangle=$

S56.

$655^{\prime}$,

เ6ช

${ }_{8989}^{679}=$

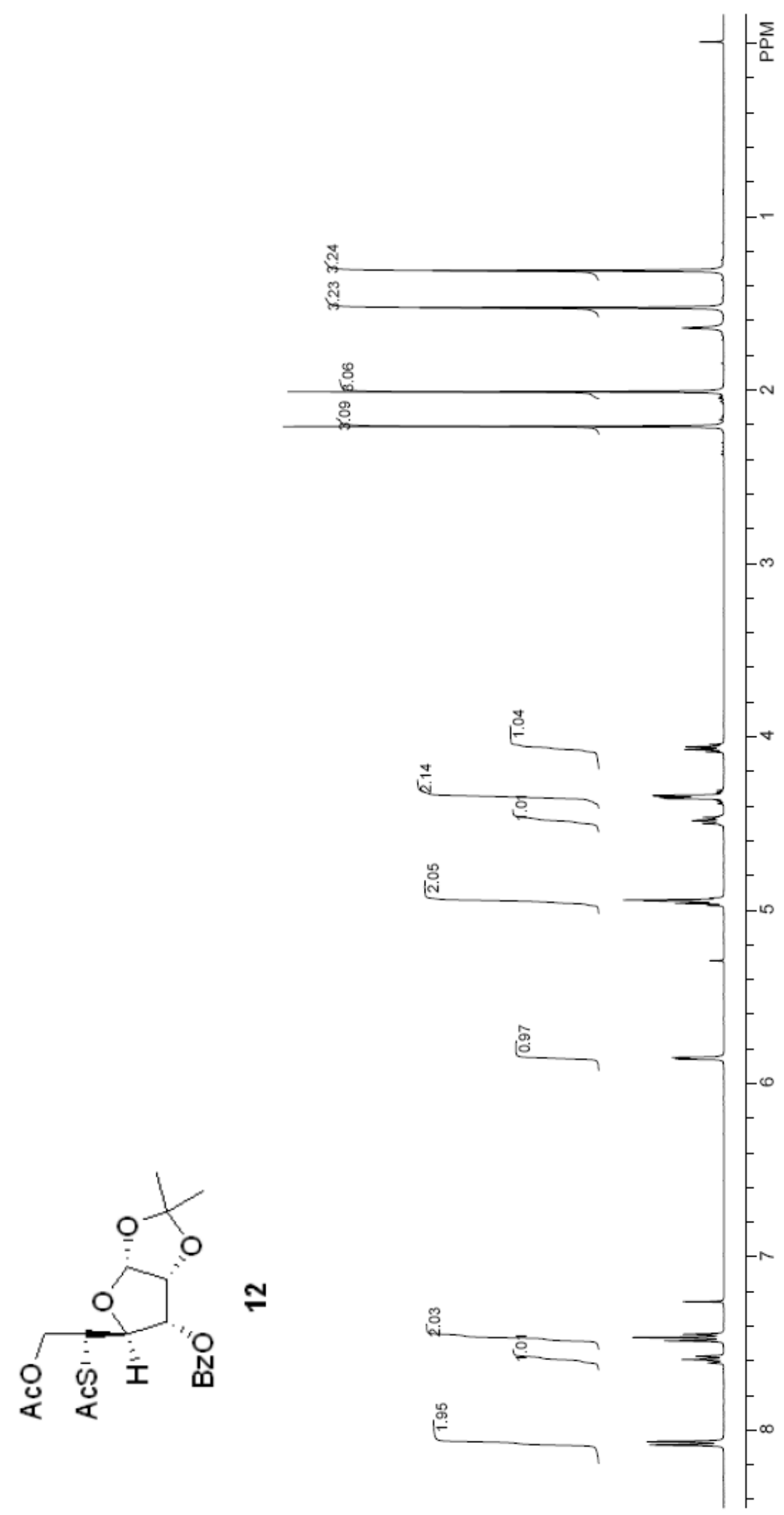

$952<=$

${ }_{9862}^{902} 2=$

${ }^{\top L S S L} \mathrm{Z}$

${ }_{190}^{890}:=$

O

980 

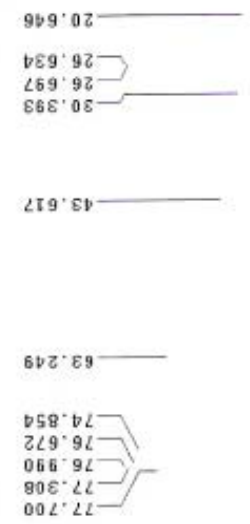

$500^{\circ} 60 \mathrm{~T}$

$052 \cdot 811$

$6 \angle t^{-} 82 T$

$62 T^{\circ} 82 \mathrm{I}$
$\angle 9 \mathrm{Q}^{\circ} 62 \mathrm{~T}$

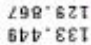

tน6. $99 T$

$88 E^{\circ} 0 \angle \mathrm{I}$

SEs. $861-$

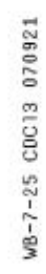

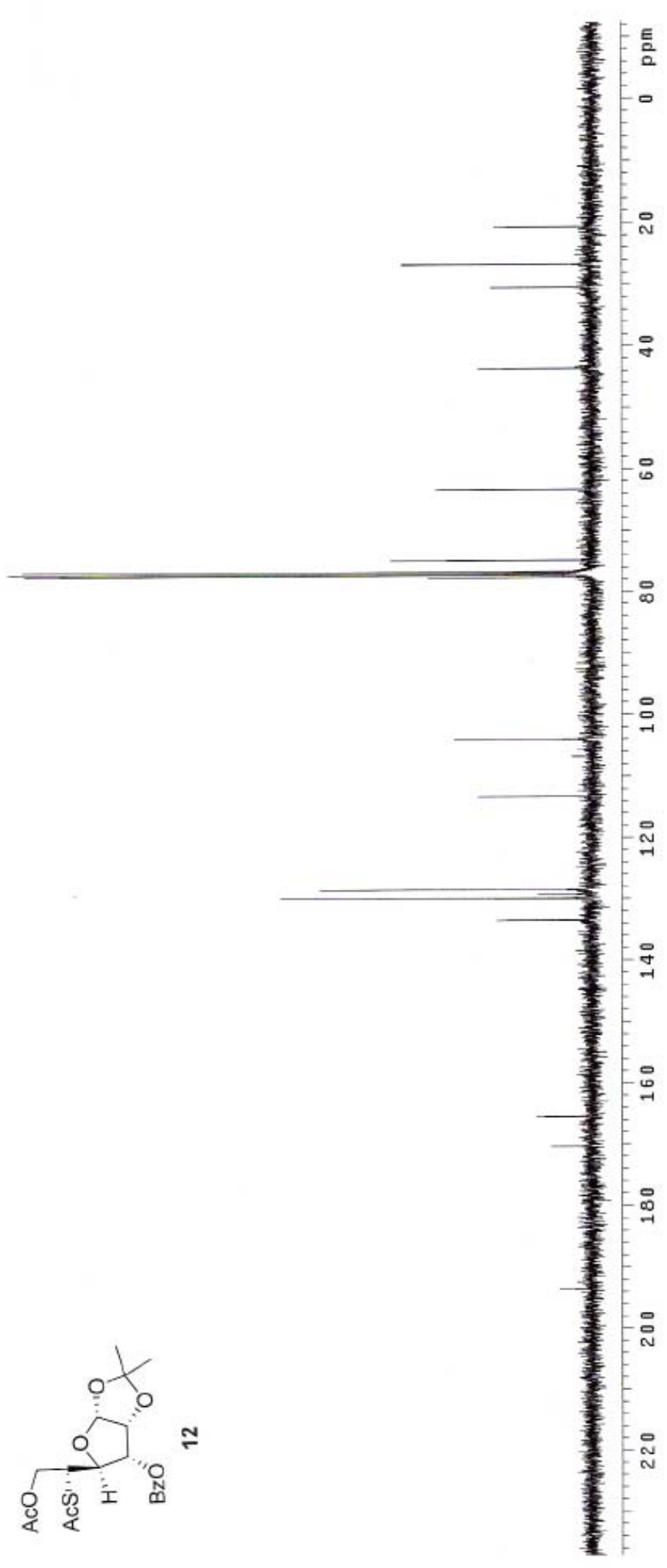


$89 Z^{\prime}$
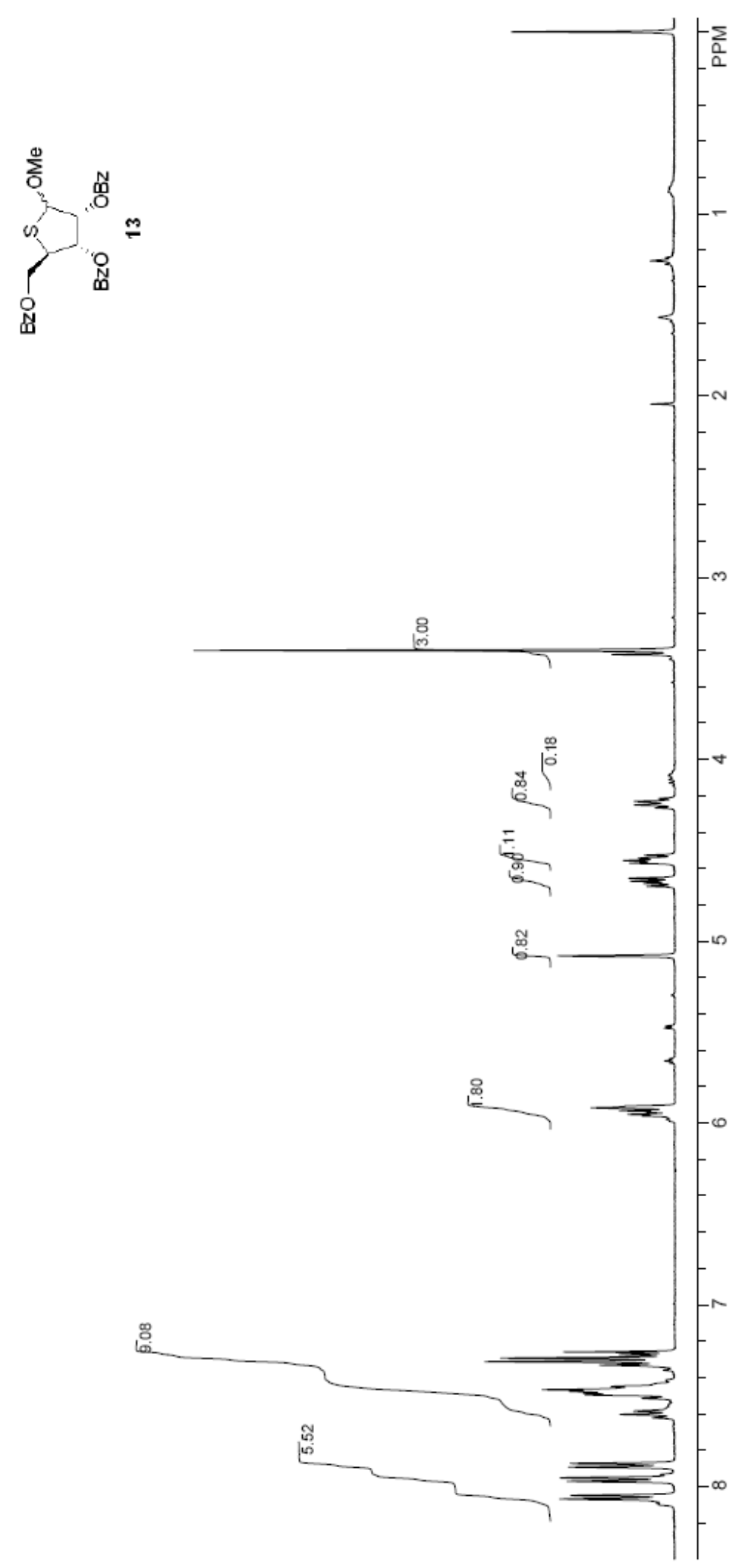

电活:

076.5
256.5
$196 \%$

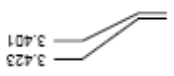

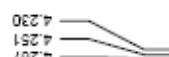

${ }_{20 \tau ;}^{2 a r y}$

${ }_{99 S \%}=$

1297
8997

6997

$2697=$

080 '

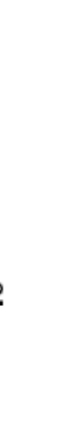




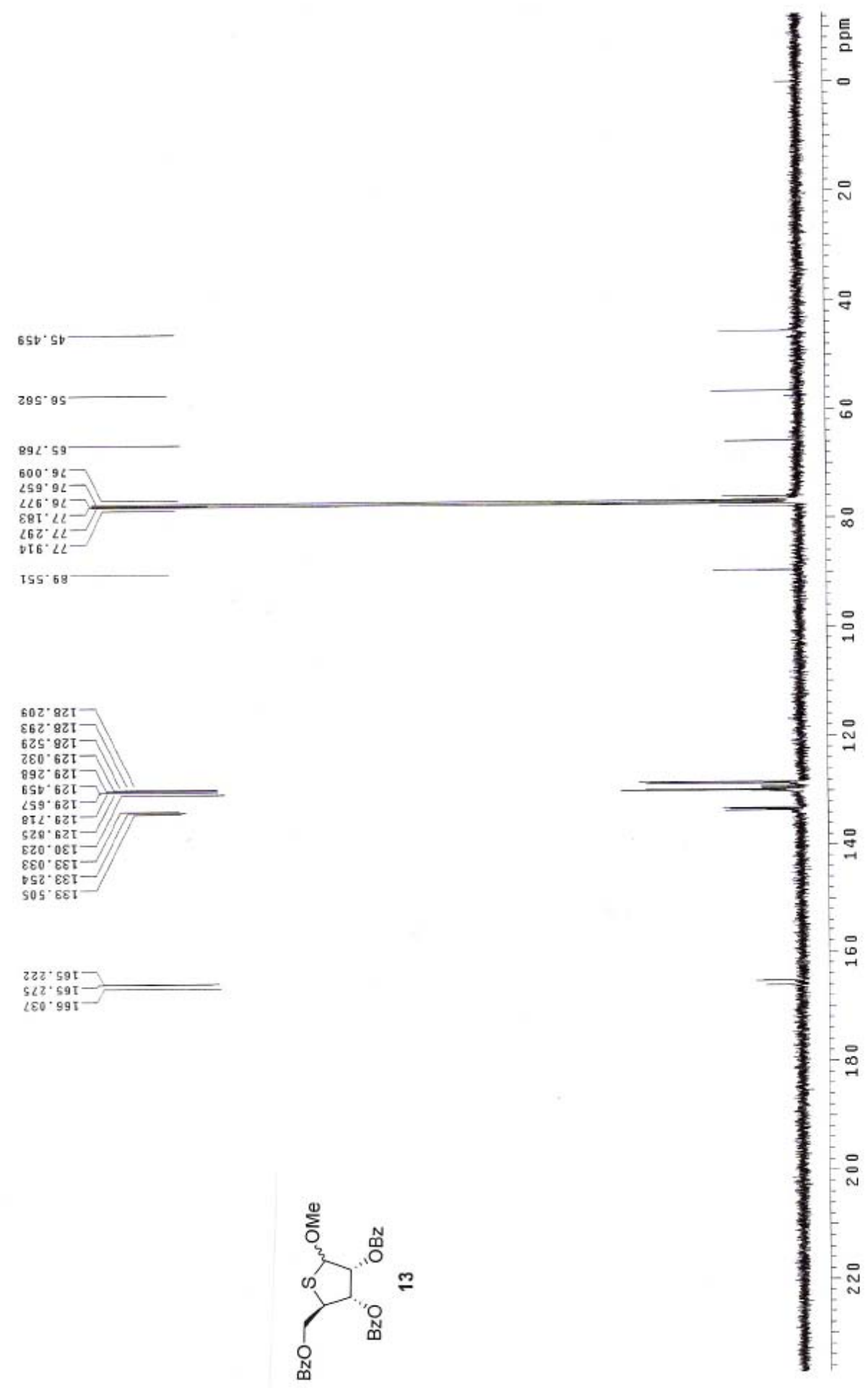




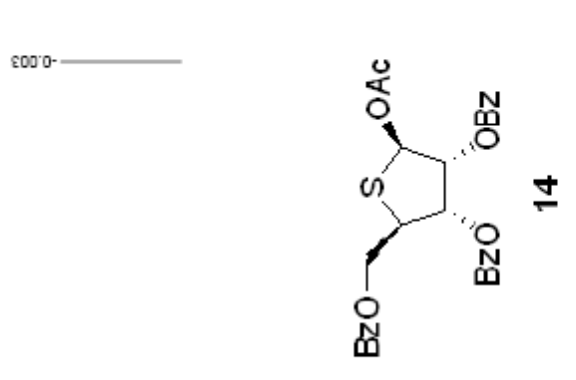

28s'เ

$64 \mathrm{z}$

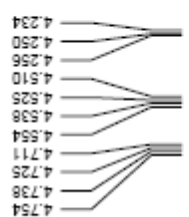

s.
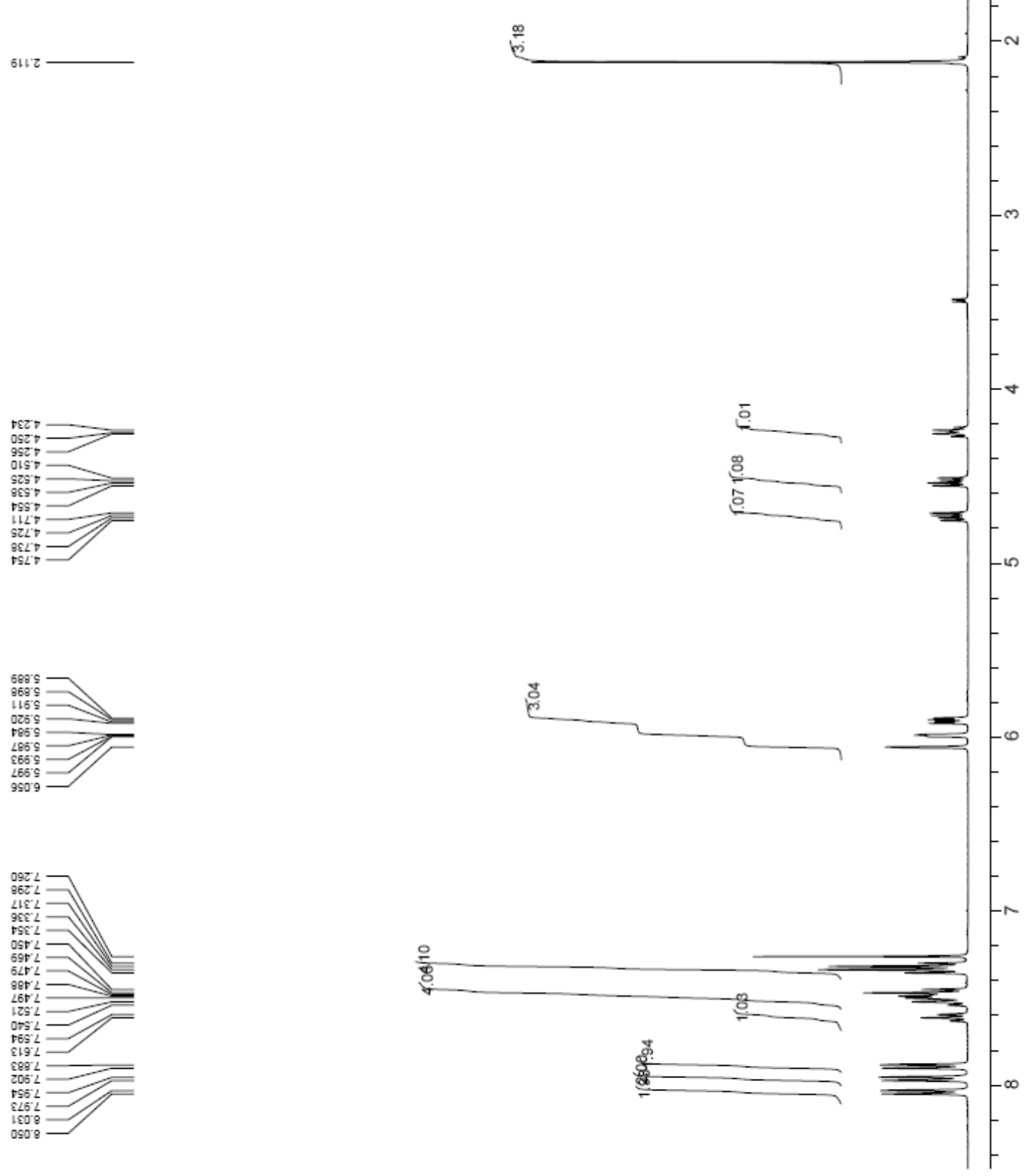
D98.0Z

$9 b T^{\prime} 9 b-$

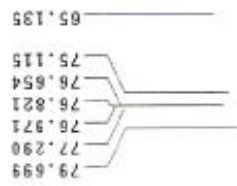

$092.821-$

$89 E^{\circ} 821-$
$995^{\circ} 821-$

$995^{\circ} 82 \mathrm{I}-$
$918^{\circ} 8 \mathrm{II}-$

$\triangle E E^{\circ}-92 \mathrm{~T}$

$668^{\circ} 62 \mathrm{~T}$

$96 L^{\circ} 62 \mathrm{I}$

$098^{\circ} 621$

$2 \varepsilon t^{\circ} \varepsilon \varepsilon t$

$286^{\circ} .691$

$558^{\circ} 59 \mathrm{I}$
$206^{\circ} 59 \mathrm{I}-$
$225^{\circ} 69 \mathrm{~T}$

$\angle 25^{\circ} 69 \mathrm{~T}$

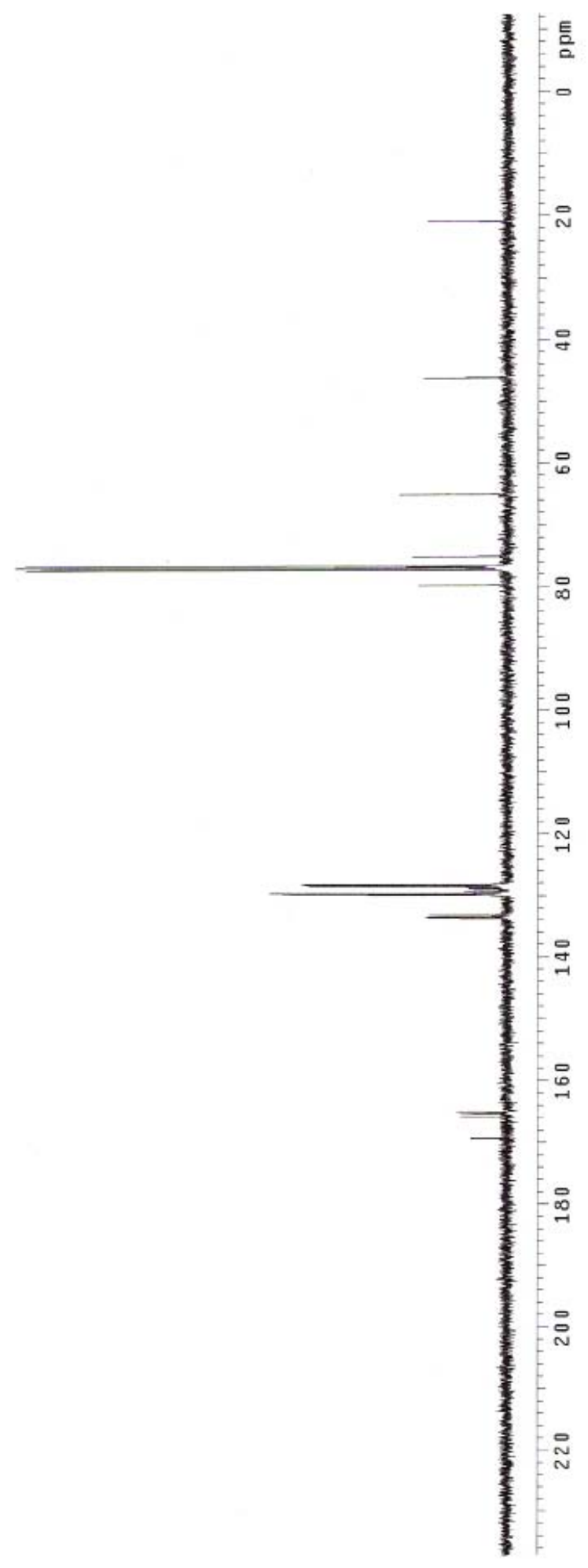




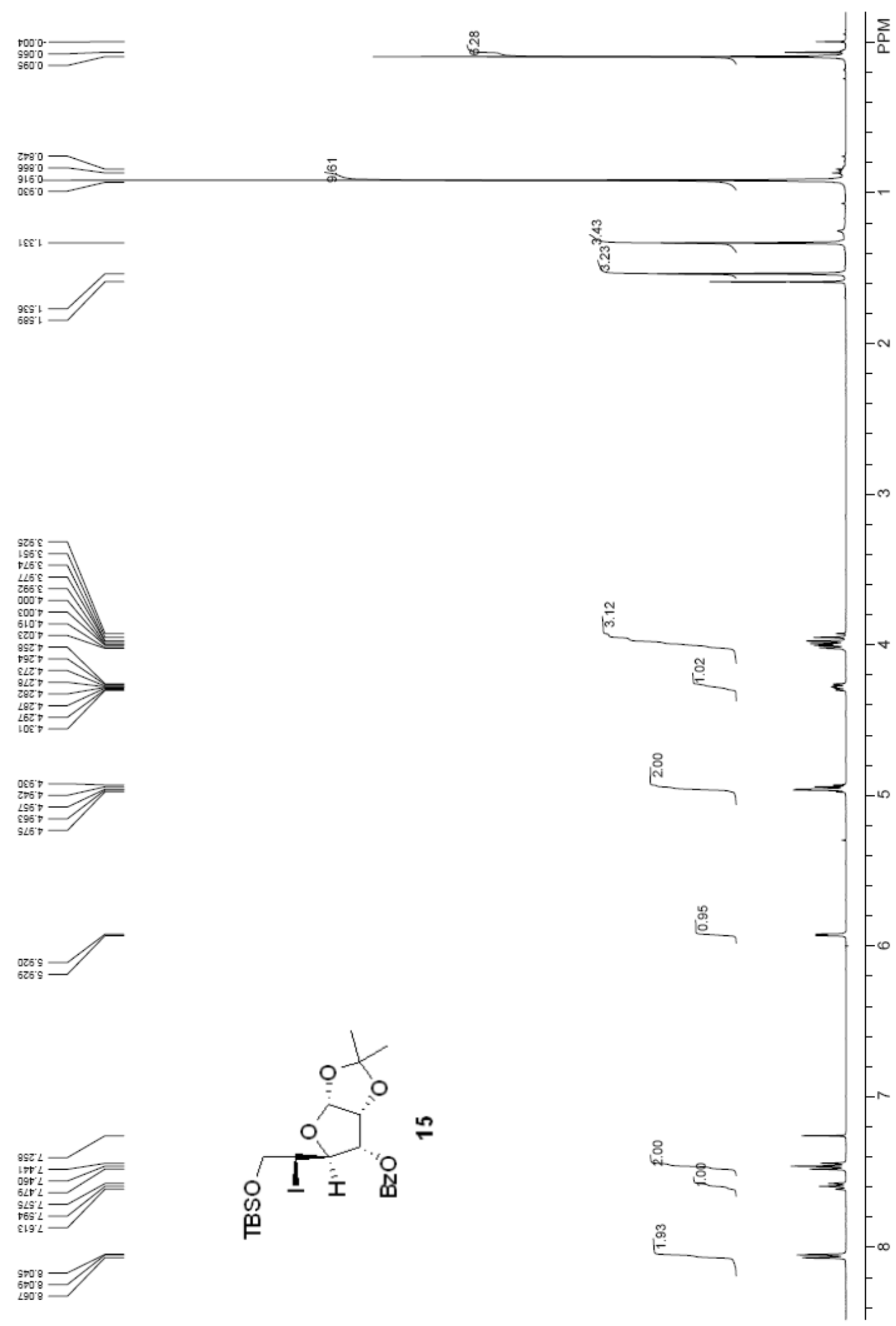


$588^{\circ} \cdot 5-$

b6 t. $8 \mathrm{~T}$

$89 L^{\circ} \mathrm{sz}$

$099^{\circ} .92-$
$828^{\circ} .92$

$585^{\circ} 98^{-}$

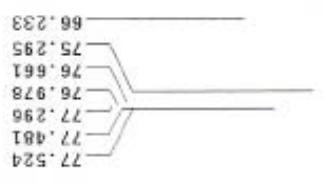

G6S' $b 0 \mathrm{I}$

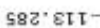

725.821

$\angle 98^{\circ} 62 \mathrm{~T}$
$868^{\circ} 8 \mathrm{I}^{\circ}$

99. 59 T

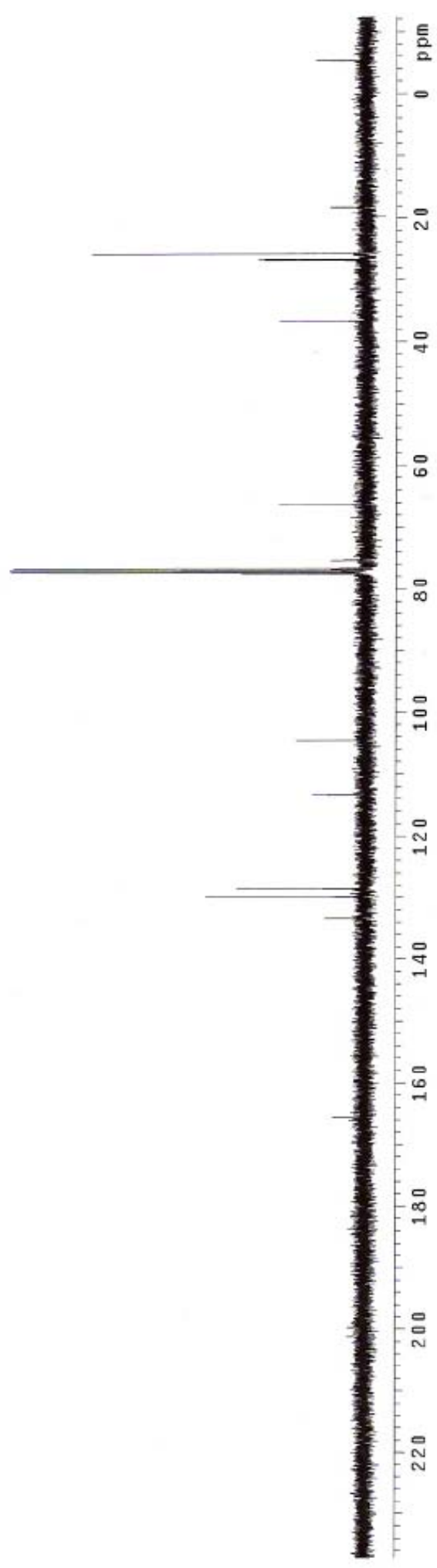


ع900

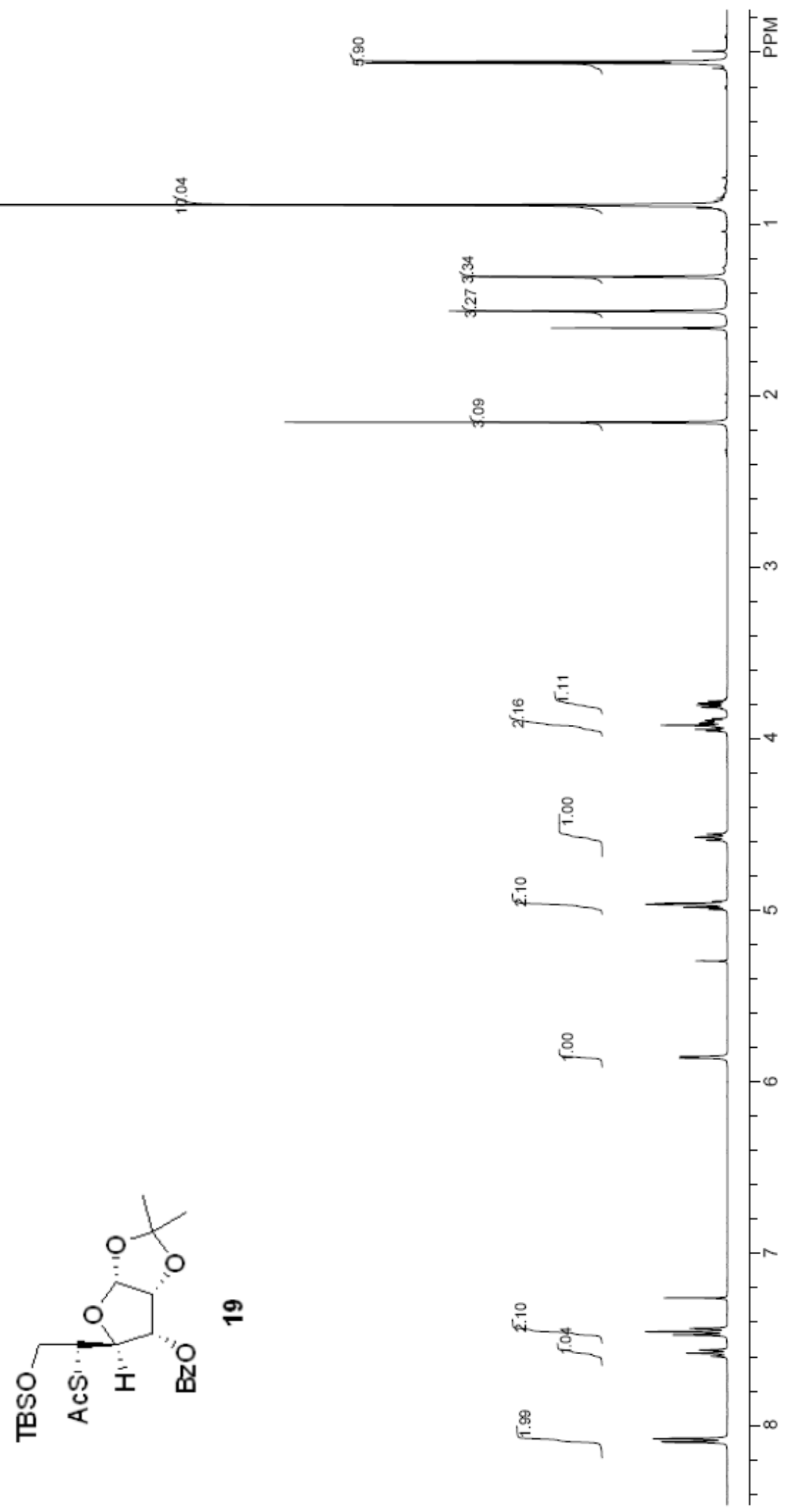

toเ'

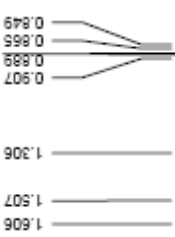

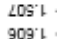

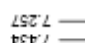

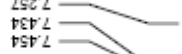

$\frac{\varepsilon<V^{2}}{\log _{9} \leq 2}=\ldots$

${ }_{62 S} S^{\prime} L$

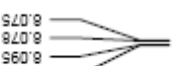

9200
4600

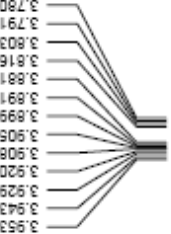

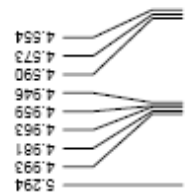

$\frac{0989}{6989}$

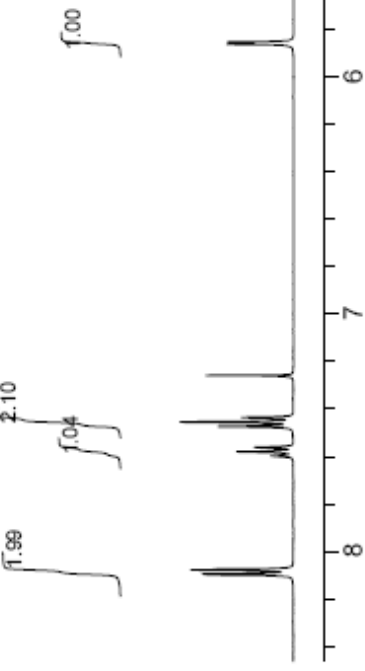


s95.s--

ISt:S-

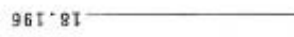

$192.52-$

$\angle 85.92$

$\angle 0 \% \cdot 08$

$B S t \cdot 2 b$

$202 \cdot 29$

$585 \cdot 52$

$185^{\circ} \cdot 9 L-$
$859 \cdot 9 L-$

$5 \angle 6 \cdot 92-$

$998^{\circ} \angle L$

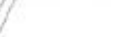

$696^{\circ} 80 \mathrm{I}$

$090^{\circ} 8 \mathrm{II}$

$49.821-$

$\begin{aligned} 3 \\ 605\end{aligned} .621-$

$305.621-$

$25 V^{\circ} 59 T^{-}$

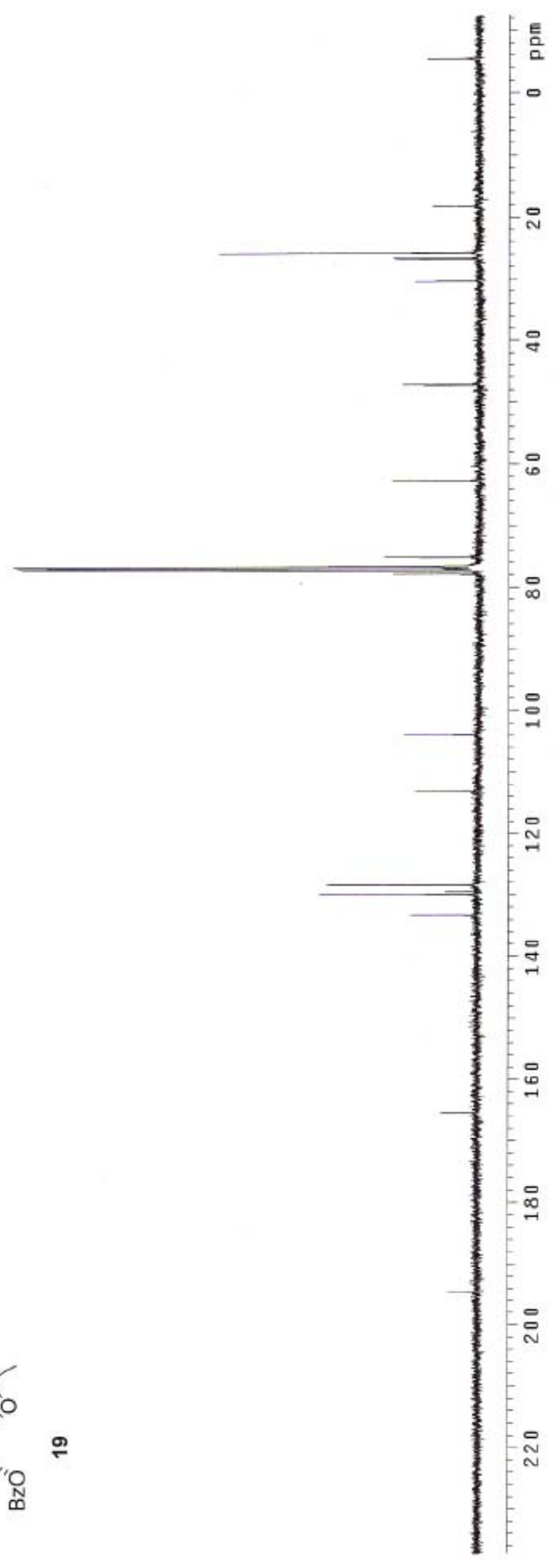




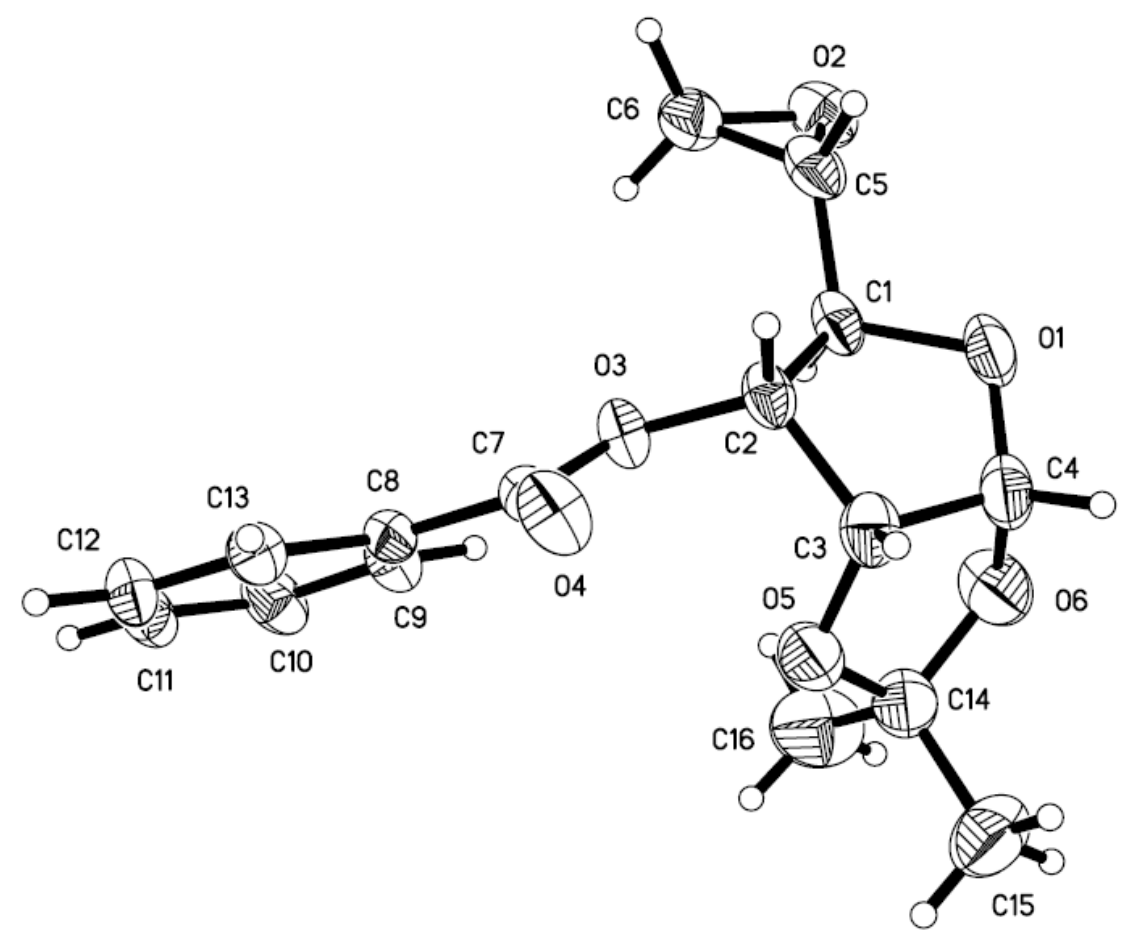

ORTEP drawing for compound $\mathbf{9}$. 


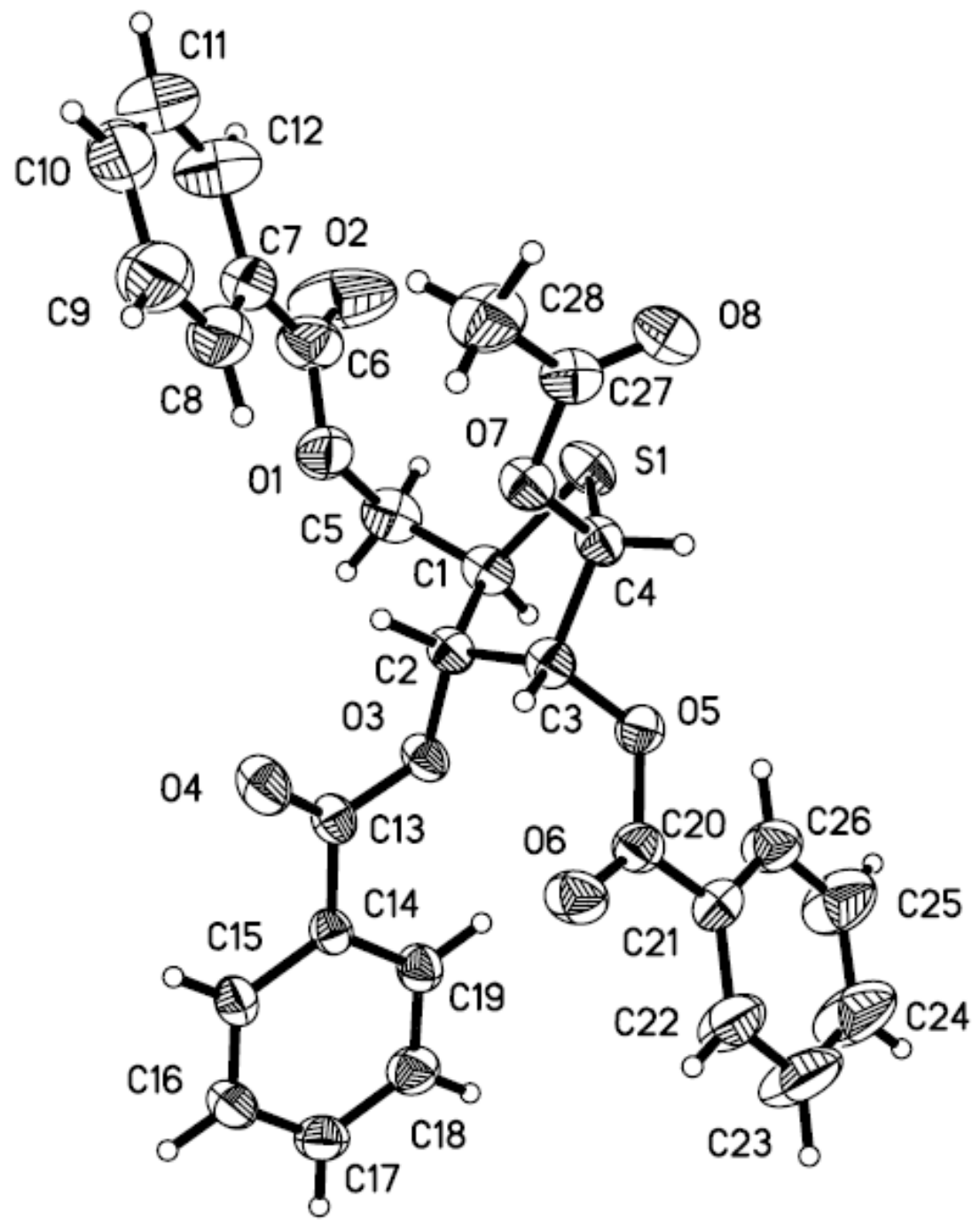

ORTEP drawing for compound 14. 